\title{
Diverticular Disease: a Gut Microbiota Perspective
}

\author{
Andrea Ticinesi ${ }^{1,2}$, Antonio Nouvenne ${ }^{1,2}$, Vincenzo Corrente ${ }^{1}$, Claudio Tana ${ }^{1}$, Francesco Di Mario ${ }^{1,3}$, Tiziana Meschi ${ }^{1,2,3}$
}

1) Geriatric Rehabilitation

Department, University-

Hospital of Parma, Parma

2) Microbiome Research Hub,

University of Parma, Parma

3) Department of Medicine and Surgery, University of Parma,

Parma, Italy
Address for correspondence: Andrea Ticinesi, MD, $\mathrm{PhD}$

Geriatric-Rehabilitation

Department, Parma

University-Hospital (Azienda

Ospedaliero-Universitaria di

Parma), via Antonio Gramsci

14, 43126 Parma, Italy.

aticinesi@ao.pr.it

Received: 13.05.2019

Accepted: 23.07.2019

\begin{abstract}
Gut microbiota composition and functionality are involved in the pathophysiology of several intestinal and extraintestinal diseases, and are increasingly considered a modulator of local and systemic inflammation. However, the involvement of gut microbiota in diverticulosis and in diverticular disease is still poorly investigated. In this review, we critically analyze the existing evidence on the fecal and mucosa-associated microbiota composition and functionality across different stages of diverticular disease. We also explore the influence of risk factors for diverticulosis on gut microbiota composition, and speculate on the possible relevance of these associations for the pathogenesis of diverticula. We overview the current treatments of diverticular disease targeting the intestinal microbiome, highlighting the current areas of uncertainty and the need for future studies. Although no conclusive remarks on the relationship between microbiota and diverticular disease can be made, preliminary data suggest that abdominal symptoms are associated with reduced representation of taxa with a possible anti-inflammatory effect, such as Clostridium cluster IV, and overgrowth of Enterobacteriaceae, Bifidobacteria and Akkermansia. The role of the microbiota in the early stages of the disease is still very uncertain. Future studies should help to disentangle the role of the microbiome in the pathogenesis of diverticular disease and its progression towards more severe forms.
\end{abstract}

Key words: diverticulosis - microbiome - mucosa-associated microbiome - fecal microbiota - metagenomics - acute diverticulitis - dysbiosis - diet - constipation.

Abbreviations: IBD: inflammatory bowel disease; SCAD: segmental colitis associated with diverticulosis; SUDD: symptomatic uncomplicated diverticular disease.

\section{INTRODUCTION}

Diverticulosis is a chronic condition implying the herniation of colonic mucosa and submucosa through the muscle layer at points of weakness of the intestinal wall, forming a protrusion of the gut lumen [1]. This asymptomatic condition may evolve into a clinically significant diverticular disease, with manifestations ranging from persistent abdominal symptoms without evidence of colonic inflammation (Symptomatic Uncomplicated Diverticular Disease, SUDD) to diverticulitis or Segmental Colitis Associated with Diverticulosis (SCAD) $[1,2]$.
While in the early stages the pathogenesis of diverticula is mainly conditioned by mechanical factors, later the inflammation and ischemia of the intestinal mucosa play a pivotal role in the development of diverticular disease and diverticulitis [1]. Several risk factors, ranging from diet to altered colonic motility, have been identified, but the precise pathophysiological cascade leading to a clinically relevant disease remains still uncertain [2].

Recent studies have focused on the possible role of the gut microbiota. The emergence of culture-independent methods of microbial profiling has in fact improved the understanding of the extreme complexity of both fecal and mucosa-associated microbiota [3, 4], highlighting its fundamental role in the onset and regulation of inflammation [5]. The pathophysiological role of the intestinal microbiota has been extensively studied for other gastrointestinal diseases, such as inflammatory bowel disease (IBD) [6] and colon cancer [7, 8], and even for extraintestinal diseases, including metabolic syndrome [9], neurodegenerative diseases [10], kidney stones [11] and muscle-wasting disorders $[12,13]$. Dysbiosis, defined as a reduced microbiome biodiversity possibly associated with 
imbalances between symbionts and pathobionts, is rapidly emerging as a fundamental biomarker of health status and an active modulator of complex pathophysiological processes [14], including a two-way interaction with the immune system [15]. Dysbiosis represents a generic alteration of the healthy gut microbiota homeostasis, and implies different characteristics and microbiome compositions in different diseases.

The role of the intestinal microbiome in diverticular disease, one of the most common gastroenterological disorders with prevalence up to $20 \%$ in subjects undergoing colonoscopy [2], is still uncertain. The characteristics of the microbiota dysbiosis, detected in other gastrointestinal diseases, such as IBD, cannot be transferred to diverticular disease. The interaction between environmental risk factors and the microbiome in determining the progression from diverticulosis to SUDD, SCAD or diverticulitis, though hypothesized by several authors [16-21], is also debated. Thus, the aim of the present review is to critically summarize the current literature state-of-art on the association between microbiota and diverticular disease, providing suggestions of possible areas of development for research and clinical practice in the future.

\section{PROFILING THE MICROBIOTA IN DIVERTICULAR DISEASE}

\section{Studies on fecal microbiota}

At the current state-of-art, the fecal microbiota in patients with diverticulosis or diverticular disease has been profiled in only seven studies, with conflicting results (Table I).
Kvasnovsky et al. [22] analyzed stool samples from 28 patients with SUDD by means of $16 \mathrm{~S}$ rRNA microbial profiling. They found that a higher bloating severity score was associated with the relative abundance of Ruminococcus (in a positive way) and Roseburia (in a negative way), while the intensity of pain was significantly correlated with the relative abundance of Cyanobacterium [22]. An individual history of episodes of acute diverticulitis was associated with an overgrowth of Pseudobutyrivibrio, Bifidobacterium and Christensenellaceae family, and the overall microbiome biodiversity was positively correlated with fecal calprotectin, an index of gut mucosa inflammation [22]. Although limited by the absence of a healthy control group and low sample size, these results suggest that specific fecal microbiome changes mirror the severity of symptoms and the level of inflammation, but do not allow to draw conclusions on the active involvement of microbiota in the pathophysiological process.

In a small series of four patients with diverticular disease, Ponziani et al. detected an increased abundance of Roseburia, Veillonella, Haemophilus and Streptococcus prior to treatment [23]. These dysbiotic landmarks are different from those typically seen in IBD, where reduced abundance of Roseburia is strongly associated with disease activity scores [24].

In another study, Barbara et al. [25] analyzed fecal samples from 16 subjects with asymptomatic diverticulosis, 8 patients with SUDD and 14 controls without diverticulosis. The overall fecal microbiota composition consistently overlapped among the three groups, but subjects with diverticula had a significantly lower abundance of Clostridium cluster IV than controls,

Table I. Studies profiling the fecal microbiome in diverticular disease.

\begin{tabular}{|c|c|c|c|}
\hline $\begin{array}{l}\text { Author [ref] (year } \\
\text { and study type) }\end{array}$ & Participants & $\begin{array}{l}\text { Type of analyses } \\
\text { performed }\end{array}$ & Core findings \\
\hline $\begin{array}{l}\text { Kvasnovsky }[22] \\
(2017, \text { cross- } \\
\text { sectional) }\end{array}$ & 28 patients with SUDD & $\begin{array}{l}\text { Metagenomics (16S rRNA } \\
\text { microbial profiling) }\end{array}$ & $\begin{array}{l}\text { Bloating severity score and previous diverticulitis correlated with the } \\
\text { abundance of some taxa } \\
\text { Alpha diversity correlated with fecal calprotectin }\end{array}$ \\
\hline $\begin{array}{l}\text { Ponziani } \\
{[23](2016,} \\
\text { intervention) }\end{array}$ & 4 patients with SUDD & $\begin{array}{l}\text { Metagenomics ( } 16 \mathrm{~S} \text { rRNA } \\
\text { microbial profiling) }\end{array}$ & $\begin{array}{l}\text { The presence of SUDD is associated with increased representation of } \\
\text { Roseburia, Veillonella, Haemophilus and Streptococcus } \\
\text { These alterations are attenuated by rifaximin treatment }\end{array}$ \\
\hline $\begin{array}{l}\text { Barbara }[25] \\
(2017, \text { case- } \\
\text { control) }\end{array}$ & $\begin{array}{l}8 \text { patients with SUDD } \\
16 \text { patients with } \\
\text { diverticulosis } \\
14 \text { controls }\end{array}$ & $\begin{array}{l}\text { Metagenomics (16S rRNA } \\
\text { microbial profiling) } \\
\text { Metabolomics (1H NMR) }\end{array}$ & $\begin{array}{l}\text { Patients with SUDD and, to lower extent, diverticulosis showed depletion of } \\
\text { taxa with anti-inflammatory properties } \\
\text { Metabolomic analyses showed a different microbiome functionality in } \\
\text { patients with DD }\end{array}$ \\
\hline $\begin{array}{l}\text { Daniels [29] } \\
\text { (2014, case- } \\
\text { control) }\end{array}$ & $\begin{array}{l}31 \text { patients with } \\
\text { uncomplicated } \mathrm{AD} \\
25 \text { controls }\end{array}$ & PCR-based profiling & $\begin{array}{l}\text { Higher abundance of Proteobacteria, and particularly Enterobacteriaceae, in } \\
\text { patients than in controls } \\
\text { No differences in the Bacteroidetes/Firmicutes ratio }\end{array}$ \\
\hline $\begin{array}{l}\text { Tursi }[30](2016 \\
\text { case-control) }\end{array}$ & $\begin{array}{l}15 \text { patients with SUDD } \\
13 \text { patients with } \\
\text { diverticulosis } \\
16 \text { controls }\end{array}$ & $\begin{array}{l}\text { RT-PCR on targeted } \\
\text { microorganisms } \\
\text { Metabolomics (1H NMR) }\end{array}$ & $\begin{array}{l}\text { Little differences in microbiome composition across groups } \\
\text { Akkermansia more represented in patients than controls } \\
\text { Lower N-acetyl compound fecal levels in patients than controls }\end{array}$ \\
\hline $\begin{array}{l}\text { Laghi }[31](2018, \\
\text { intervention) }\end{array}$ & $\begin{array}{l}13 \text { female patients with } \\
\text { SUDD }\end{array}$ & $\begin{array}{l}\text { RT-PCR on targeted } \\
\text { microorganisms } \\
\text { Metabolomics (1H NMR) }\end{array}$ & $\begin{array}{l}\text { Little differences in microbiome composition across groups } \\
\text { Akkermansia more represented in patients than controls }\end{array}$ \\
\hline $\begin{array}{l}\text { Lopetuso [34] } \\
(2017, \text { case- } \\
\text { control) }\end{array}$ & $\begin{array}{l}4 \text { patients with DD } \\
8 \text { controls }\end{array}$ & $\begin{array}{l}\text { Metagenomics ( } 16 \mathrm{~S} \text { rRNA } \\
\text { microbial profiling) }\end{array}$ & $\begin{array}{l}\text { Similar fecal microbiota composition between patients and controls, except } \\
\text { for a Bacteroides fragilis depletion in patients }\end{array}$ \\
\hline
\end{tabular}

SUDD: Symptomatic Uncomplicated Diverticular Disease; AD: Acute Diverticulitis; DD: Diverticular Disease; PCR: Polymerase-Chain Reaction; NMR: Nuclear Magnetic Resonance; RT-PCR: Real-Time Polymerase-Chain Reaction. 
while patients with SUDD had also a reduced abundance of Fusobacterium and Lactobacillaceae [25]. Interestingly, both Clostridium cluster IV and Lactobacillaceae have been associated with marked anti-inflammatory and immuneregulatory effects in experimental models of colitis [26-28]. This circumstance suggests that selective depletion of such taxa in the fecal microbiota may be involved in the development of SUDD, although the cross-sectional design of the Barbara et al. study [25] does not allow to draw such conclusions.

Increasing severity of inflammation in SUDD may also be associated with a higher fecal microbiota biodiversity. This concept is supported by the findings of a cross-sectional study where fecal samples from 31 patients suffering from acute diverticulitis and 25 healthy controls were compared by a polymerase-chain reaction (PCR) [29]. Although limited by the absence of a comprehensive metagenomics profiling of fecal microbial communities, this study showed a significant overgrowth of Enterobacteriaceae in stools of patients with diverticulitis, suggesting that the inflammatory process and its complications may be associated with a specific imbalance between symbionts and pathobionts [29]. The physiopathological cascade from asymptomatic diverticulosis to acute diverticulitis seems to be accompanied by increasing degrees of microbiota homeostasis disruption.

However, there are also studies showing little, if no, modification in the fecal microbiota composition of patients with diverticular disease. Tursi et al. [30] analyzed stool samples from 15 patients with SUDD, 13 subjects with asymptomatic diverticulosis and 16 controls using a PCR-based approach. They identified no differences in the abundance of the main microbiome components across the three groups, except for Akkermansia muciniphila, which showed an increasing abundance trend in SUDD [30]. This trend was associated with a different fecal metabolomics profile, including lower $\mathrm{N}$-acetyl compound and isovalerate levels in SUDD [30]. The increased abundance of Akkermansia in the fecal samples of patients with SUDD was also confirmed in another pilot study testing the impact of pharmacologic and probiotic treatment in a small group of patients [31]. Interestingly, these characteristics of gut microbiota dysbiosis in SUDD are different than those detected in IBD, where the presence of Akkermansia is generally reduced, and not increased [32].

These circumstances suggest a different functionality of the intestinal microbiome in SUDD, while the SUDDassociated Akkermansia overgrowth may either be the result of an increased mucin production or a homeostatic response against inflammation. Akkermansia has been associated with anti-inflammatory properties, but its growth is also stimulated by colonic inflammation itself, using mucin as a metabolic substrate [33].

Diverticular disease has been associated with the depletion of Bacteroides fragilis, Collinsella aerofaciens and Collinsella stercoris in another small study comparing the fecal microbiome of 4 patients and 8 controls, but no significant differences in the abundance of the main bacterial species could be detected [34]. However, Bacteroides fragilis could play a role in the modulation of inflammation [35], and this finding could have a pathophysiological relevance in spite of the very small sample size of the study.

\section{Studies on the mucosa-associated microbiota}

Since the emergence of high-throughput metagenomics sequencing techniques in the 2000s, the composition of fecal microbiota has been considered as a valid proxy of the complex microbial communities harbored in the intestinal lumen [3]. However, recent studies have raised several doubts on this assumption.

First, the fecal microbiota composition is highly dependent on stool consistency and number of bowel movements even in subjects without constipation [36]. The fecal microbial load is inversely associated with stool moisture, so that the differences in absolute bacterial counts between patients with constipation and patients with diarrhea may not be adequately captured by fecal metagenomics [37]. Second, studies comparing fecal microbiota with mucosa-associated microbiota, determined on biopsy samples collected during colonoscopy in the same individuals have shown that the overlap is only partial $[37,38]$. In other terms, mucosa-associated microbiota may be different from fecal microbiota, and represent a more reliable picture of the microbial communities involved in the pathophysiology of gastrointestinal diseases $[38,39]$.

In diverticular disease, the analysis of mucosa-associated microbiota may thus have much more relevance than fecal microbiota [40]. Diverticular disease generally affects only limited segments of the colon, which may harbor different microbial communities than segments spared by the disease. Moreover, the diverticular pockets may represent unique niches selectively promoting the development of specific microbial communities which can play a role in the progression of diverticulosis towards symptomatic disease [40].

In fact, PCR analyses of colonic mucosal biopsies from 16 patients with diverticular disease showed a significant overrepresentation of Enterobacteriaceae than biopsies of 35 controls without diverticula [41]. Blooming of Enterobacteriaceae is one of the main distinctive features of severe dysbiosis, and generally represents the consequence of mucosal inflammation $[42,43]$. These results were confirmed in 9 patients with SCAD, who were subdued to multiple colonic biopsies from diseased tissue and adjacent colonic mucosa [44]. Bacterial 16S rRNA microbial profiling revealed that the mucosal microbiota was significantly different between diseased and adjacent tissue, but also that Enterobacteriaceae, such as Pseudomonas, were overrepresented in all samples [44]. Another small study revealed that acute diverticulitis was associated with the overrepresentation of Bifidobacteria, namely Bifidobacterium longum [45]. This finding confirms the presence of a deep disruption of the mucosa-associated microbial communities, even though Bifidobacterium longum has been associated with anti-inflammatory properties for animal models of colitis [46] and human enterocytes [47]. Thus, the bifidobacterial expansion might be interpreted as a homeostatic mechanism, but should be confirmed in larger studies with a comprehensive profiling of the mucosal microbiota.

In the above referenced Barbara et al. study [25], metagenomics profiling of mucosa-associated microbiota was also performed. No significant differences could be detected between controls and subjects with asymptomatic diverticulosis, except for a reduced Enterobacteriaceae 
abundance in cases [25]. Biopsies of patients with SUDD taken in the diverticular region showed significantly reduced representation of Akkermansia, while the mucosal macrophage count was negatively correlated with Clostridium cluster IV abundance and positively correlated with the abundance of Fusobacterium [25]. Although conflicting with the results of previous studies, these findings support the involvement of gut microbiota in the transition from asymptomatic diverticulosis to diverticular disease, and suggest an interplay between mucosa-associated microbial communities and the local immune system.

However, in the largest study to date, comparing mucosaassociated microbiota from 226 subjects with diverticulosis and 309 healthy controls, the results were substantially negative [48]. Apart from a mild increase in bacterial biodiversity and abundance of the phylum Proteobacteria and the family Comamonadaceae, the mucosa-associated microbiota composition substantially overlapped between cases and controls, irrespective of the number of diverticula detected by colonoscopy [48]. It is noteworthy that only subjects with asymptomatic diverticulosis were enrolled in this study, and comparison with patients with SUDD or diverticulitis was lacking.

An overview of the existing studies where profiling of the mucosa-associated microbiota in diverticular disease or diverticulosis was performed is presented in Table II.

\section{THE MICROBIOTA IN DIVERTICULAR DISEASE: BYSTANDER OR ACTIVE PLAYER?}

The existing studies profiling fecal and mucosa-associated microbiota in diverticular disease do not permit the drawing of conclusions on the precise alterations associated with the disease (if any). The studies suffered in many cases from limitations due to a reduced sample size, laboratory techniques (PCR instead of metagenomics), insufficient clinical characterization of symptoms and disease burden, and a mixed setting of enrolment. The results were also conflicting and incoherent across different studies. However, some points of interest, which could be useful for designing future studies, emerged. First, asymptomatic diverticulosis does not seem to be associated with significant intestinal microbiome alterations. Thus, the involvement of the microbiome in the early phases of diverticulum pathogenesis seems reduced. However, some significant changes do occur in the microbiome composition when diverticulosis evolves into SCAD or acute diverticulitis: depletion of taxa with purported anti-inflammatory activity, such as Clostridium cluster IV, Lactobacilli and Bacteroides, Conversely, overgrowth of Bifidobacteria, Enterobacteriaceae and Akkermansia seem to occur in diverticular disease, but its significance is uncertain. In fact, these taxa may exhibit both anti-inflammatory (Bifidobacteria, Akkermansia) or pro-inflammatory (Enterobacteriaceae) activity, or may represent "bystanders" growing in response to changes in the gut microenvironment, without any direct pathophysiological involvement.

These findings support the concept that microbiota could be involved in the progression of diverticulosis to diverticular disease and diverticulitis, but not in the pathogenesis of diverticula, as implicitly hypothesized by Daniels et al. [17] and Søreide et al. [49]. Thus, the microbiota may have relevance particularly in SUDD and diverticulitis, and represents also a specific therapeutical target in these stages [50].

SUDD is in fact associated with a different fecal $[25,30]$ and urinary [51] metabolomics profile than asymptomatic

Table II. Studies profiling the mucosa-associated microbiome in diverticular disease.

\begin{tabular}{|c|c|c|c|}
\hline $\begin{array}{l}\text { Author [ref] (year and study } \\
\text { type) }\end{array}$ & Participants & Type of analyses performed & Core findings \\
\hline $\begin{array}{l}\text { Linninge [41] (2018, case- } \\
\text { control) }\end{array}$ & $\begin{array}{l}16 \text { patients with DD } \\
35 \text { controls }\end{array}$ & $\begin{array}{l}\text { PCR-based profiling of } \\
\text { Enterobacteriaceae }\end{array}$ & $\begin{array}{l}\text { Mucosa-associated microbiota of patients } \\
\text { with DD exhibits higher amounts of } \\
\text { Enterobacteriaceae than controls }\end{array}$ \\
\hline $\begin{array}{l}\text { Schieffer }[44] \text { (2017, cross- } \\
\text { sectional) }\end{array}$ & $\begin{array}{l}9 \text { patients with chronic recurrent } \\
\text { diverticulitis }\end{array}$ & $\begin{array}{l}\text { Metagenomics ( } 16 \mathrm{~S} \text { rRNA } \\
\text { microbial profiling) } \\
\text { Fungal ribosomal ITS profiling }\end{array}$ & $\begin{array}{l}\text { The microbiota associated with diseased } \\
\text { mucosa exhibits different composition } \\
\text { (overrepresented Microbacteriaceae and } \\
\text { Basidiomycota) than microbiota associated } \\
\text { with adjacent tissue }\end{array}$ \\
\hline $\begin{array}{l}\text { Gueimonde [45] (2007, } \\
\text { cross-sectional) }\end{array}$ & $\begin{array}{l}9 \text { patients with } \mathrm{AD} \\
23 \text { patients with other GI } \\
\text { conditions }\end{array}$ & Qualitative and quantitative PCR & $\begin{array}{l}\text { Overexpression of Bifidobacterium longum in } \\
\text { the mucosa of patients with } \mathrm{AD}\end{array}$ \\
\hline $\begin{array}{l}\text { Barbara }[25](2017, \text { case- } \\
\text { control) }\end{array}$ & $\begin{array}{l}8 \text { patients with SUDD } \\
16 \text { patients with diverticulosis } \\
14 \text { controls }\end{array}$ & $\begin{array}{l}\text { Metagenomics ( } 16 \mathrm{~S} \text { rRNA } \\
\text { microbial profiling) }\end{array}$ & $\begin{array}{l}\text { No differences in the microbiome } \\
\text { composition between patients with } \\
\text { diverticulosis and controls } \\
\text { Lower abundance of Akkermansia in SUDD } \\
\text { Higher abundance of Enterobacteriaceae in } \\
\text { biopsies taken in diverticula than in distant } \\
\text { sites }\end{array}$ \\
\hline $\begin{array}{l}\text { Jones }[48](2018 \text {, case- } \\
\text { control) }\end{array}$ & $\begin{array}{l}226 \text { patients with diverticulosis } \\
309 \text { controls }\end{array}$ & PCR plus metagenomics & $\begin{array}{l}\text { No substantial differences between patients } \\
\text { with diverticulosis and controls, except } \\
\text { for Proteobacteria and Comamonadaceae, } \\
\text { underrepresented in cases }\end{array}$ \\
\hline
\end{tabular}

DD: Diverticular Disease; SUDD: Symptomatic Uncomplicated Diverticular Disease; AD: Acute Diverticulitis; PCR: Polymerase-Chain Reaction; ITS: Internal Transcribed Spacer. 
diverticular disease, suggesting different microbiome functionality in this condition. Acute alterations of intestinal microbial communities, such as those induced by surgery, may play a role in the complications of diverticular disease [52]. Interestingly, in rare cases fecal microbiota transplantation for Clostridium difficile infection may induce diverticulitis [53], supporting a central role for microbiota in the development of the acute condition.

Recent studies also suggest that gastrointestinal diseases known to be associated with deep gut microbiota dysbiosis, such as ulcerative colitis and Helicobacter pylori infection may protect against the onset of diverticular disease $[54,55]$. Conversely, diverticular disease could represent a risk factor for autoimmune diseases, such as polymyalgia rheumatica, with gut-microbiota-induced immune dysfunction as a cross-road between the two conditions [56].

In this scenario, the role of gut microbiota in the pathophysiology of diverticular disease may be far from that of a simple bystander, engaging in a complex interplay between the intestinal mucosa and the whole organism. However, our understanding is only at the beginning, and future studies should help to disentangle these complex relationships.

\section{THE MICROBIOTA AND RISK FACTORS FOR DIVERTICULOSIS}

\section{Conceptual framework}

Although the current literature state-of-art does not support an active role of the intestinal microbiota in the pathogenesis of diverticula, the role of environmental risk factors for diverticulosis also deserves a discussion from a gut microbiota perspective. In fact, almost all the factors associated with an increased risk of diverticulosis represent active modulators of the microbiome composition [57]. Despite a consistent inter-individual variability, the intestinal microbial ecosystem is shaped by several environmental influences [58]. Acute stressors, such as antibiotic administration, generally induce transient perturbations that disappear after the exposure to stressors is over [14]. This resilience is, however, not absolute, and long-term signatures may persist, so that long-term exposure to environmental factors may induce persistent modifications in the microbiome composition [14].

Age, low fiber intake, excessive meat intake, alcohol, smoking, and exercise represent risk factors for both diverticulosis and gut microbiota dysbiosis $[2,57]$. Similarly, medical conditions such as obesity, multimorbidity and polypharmacy may also be associated with gut microbiota dysbiosis, and have at the same time a significant association with diverticula $[2,57]$.

In this regard, a role of the gut microbiota as a mediator of the pathophysiological link between risk factors and development of diverticulosis can be hypothesized. Prospective population-based studies with complete profiling of the microbiota should be performed in the future to clarify these aspects.

\section{The role of age}

Aging is associated with reduced biodiversity of the intestinal microbiome and increased inter-individual variability [58]. Resilience to stressors, such as antibiotic administration, is also reduced, leading to depletion of taxa with anti-inflammatory and purported metabolic beneficial activities, and overgrowth of pathobionts [58, 59]. These changes are emphasized in those subjects living in nursing homes, experiencing reduced mobility and with cognitive dysfunction [60-62]. In elderly subjects, prolonged hospitalization is also a consistent promotor of microbiota dysbiosis [63]. Conversely, aging in good health is generally associated with a gut microbiota profile resembling to that of younger persons [64].

The association between aging and diverticulosis is well known, and the incidence peak is after the age of $70[1,2]$. Although aging per se is not sufficient to determine the onset of diverticulosis, a role of age-related changes of gut microbiota in the pathogenesis of diverticula cannot be excluded, and should be investigated in the future. In fact, older age makes patients with diverticulosis more prone to symptomatic diverticular disease and clinically relevant complications, and the intestinal microbiome could play an important role in this circumstance [65].

Moreover, in recent years a specific subpopulation of patients with early-onset diverticular disease has emerged [66]. The incidence of acute diverticulitis in persons aged 40-49 years old has increased by $132 \%$ in the United States from 1980 to 2007 [66]. The causes of this epidemiologic phenomenon are still uncertain, but the presence of specific imbalances in the gut microbiota has been hypothesized [67]. The interplay between gut microbiota, genetic predisposition and other risk factors, such as diet, may also be involved [67].

\section{Dietary factors}

The main dietary imbalances associated with the risk of developing diverticulosis are low fiber intake and excessive red meat consumption. Both dietary regimens are associated with specific modifications of the intestinal microbiota.

In animal models, low-fiber diets are more often associated with overrepresentation of Enterobacteriaceae and Bacteroides than high-fiber diets [68]. In human beings, the habitual consumption of a low amount of fibers is associated with depletion of Clostridia, Actinobacteria [69] and all butyrateproducing taxa [70].

Fiber intake is positively correlated with fecal short-chain fatty acid concentrations, representing active metabolic mediators involved in the regulation of immune system and insulin sensitivity [71]. Insufficient fiber intake may predispose to alterations of the barrier function of the intestinal mucosa, promoting local and systemic inflammation and susceptibility to infection [72]. Conversely, a high-fiber diet is associated with increased microbiome biodiversity and stability [73], increased representation of taxa with purported beneficial metabolic activities [74], and modulation of inflammation [75].

High-protein diets generally trigger the overexpression of bacteria with protein-fermenting capacities, including many Enterobacteriaceae, Streptococcus and Bacteroides, at the expense of saccharolitic bacteria, such as Bifidobacteria and Lactobacilli [76]. These changes are independent of calorie content, but may be influenced by protein source [77]. Proteins from red meat are those associated with the maximal expansion 
of pathobionts, possibly altering the inflammatory balance in the gut [78].

Similar changes in gut microbiota composition are also triggered by alcohol consumption. In alcoholics, a marked dysbiosis, characterized by overrepresentation of Enterobacteriaceae, Prevotellaceae and Streptococcaceae, can be detected, with promotion of inflammation [78]. These effects may be partly counterbalanced by the polyphenol-induced changes of the microbiota in red wine consumers [79].

In summary, diet may play a pivotal role in shaping intestinal microbiota composition, and at the same time dietary imbalances increase the risk of diverticulosis. The role of the microbiota in this interplay is largely unknown, and should be investigated by future studies.

\section{Exercise and body composition}

Physical exercise is a powerful modulator of intestinal microbiota composition [80]. Regular exercise is associated with increased microbiome biodiversity, and optimal balance between taxa with anti-inflammatory activity and pathobionts [81]. These favorable changes seem to be independent of diet and body composition [82], and professional athletes generally exhibit a different microbiome functionality than sedentary individuals [83].

Conversely, obesity is associated with significant alterations in gut microbiota composition [84], and microbiota composition itself can influence body composition and therapeutical responses to dietary interventions [85].

Interestingly, habitual exercise is associated with a reduced risk of developing diverticular disease [86], while obesity is a well-established risk factor for it [87]. These epidemiological associations may be mediated by the effects of exercise on the gut microbiota, but this hypothesis has never been verified to date.

\section{Comorbidities and polypharmacy}

Functional constipation is a well-known risk factor for the development of diverticula $[1,2]$, being associated with relevant alterations of the intestinal microbiome. In adults, these alterations include depletion of Bacteroides, Roseburia and Coprococcus and overexpression of microbial functional metabolic pathways involved in methanogenesis and glycerol degradation [88]. In children, overgrowth of Bacteroides and Bifidobacteria has been demonstrated [89]. These modifications of microbial communities may trigger colonic dysmotility, by regulation of serotonin neurotransmitter transporters [90]. Additionally, increased consistency of feces is associated with different intestinal microbiota composition even when the diagnostic criteria for functional constipation are not satisfied [36].

In this scenario, the formation of diverticula may not be simply due to mechanical factors, but the changes of intestinal microbiota associated with constipation may also play a role, that should be investigated in future studies.

Moreover, some epidemiologic studies have established an association between other extra-intestinal comorbidities, such as diabetes and hypertension, and the risk of diverticulosis [91, 92]. The risk of complications of diverticular disease is also increased in multimorbid patients [93]. Interestingly, multimorbidity, i.e., the presence of two or more chronic diseases, has been recently associated with fecal microbiota dysbiosis in two separate studies, performed in hospitalized older patients [94] and a cohort of community-dwelling twins [95], respectively.

Multimorbidity is frequently associated with polypharmacy, i.e. the prescription of five or more drugs that patients should take chronically. Polypharmacy has been associated with increased gut microbiota dysbiosis, and this effect is emphasized when patients take drugs that may alter colon motility, such as opioids, non-steroidal inflammatory drugs and neuroleptics $[94,95]$. Since the prescription of some of these drugs has been epidemiologically associated with an increased risk of diverticulosis [96], the interconnections between drugs, microbiota and diverticulosis may deserve further investigation in the future.

\section{MICROBIOTA AND THE MANAGEMENT OF DIVERTICULAR DISEASE}

\section{Probiotics}

Although the involvement of the intestinal microbiome in the pathophysiology of diverticular disease still shows several areas of uncertainty, microbiome-targeted strategies have been investigated in the treatment of acute episodes of diverticulitis or in their prevention [97]. The use of probiotics in diverticular disease has been reviewed by different authors without conclusive evidence on their capacity to modify the disease course, due to the poor quality and heterogeneity of the studies [98-100]. Thus, the current clinical recommendations for the management of diverticular disease do not include the use of probiotics [18].

In fact, most of the randomized clinical trials have employed preparations with a single probiotic strain, usually Lactobacilli, administered for a limited period of time and with variable follow-up length [98-100]. These preparations were in some cases administered alone, while in other cases in association with rifaximin, anti-inflammatory drugs or even antibiotics that could have significantly limited their capacity to interact and modify the existing microbiome [98-100]. Furthermore, clinical outcomes were highly heterogeneous [98-100]. But most importantly, almost all the studies did not investigate the composition of the fecal or mucosa-associated microbiota, neither before nor after the intervention [98-100]. The only exception was the study by Lamiki et al. [101], who demonstrated a significant survival of the two components of the blend they studied, Bifidobacterium and Lactobacillus acidophilus, showing a possible protective effect against the recurrence of SUDD.

In summary, despite these relevant limitations, probiotic administration may be an effective adjuvant treatment for relieving symptom severity in SUDD, particularly bloating and, in some cases, abdominal pain [100]. The use of probiotic blends containing Lactobacilli with anti-inflammatory drugs (such as mesalazine or balsalazide) may be effective in maintaining symptom remission in SUDD [96]. Conversely, the role of probiotics or symbiotics in acute diverticulitis is even less defined, although a recent pilot study has given promising results with the administration of a blend composed 
of Lactobacillus salivarius, Lactobacillus acidophilus and Bifidobacterium lactis [100].

\section{Rifaximin}

Rifaximin has recently gained popularity for its possible positive influence on the intestinal microbiome. In fact, its efficacy in the treatment of SUDD and prevention of recurrent episodes of acute diverticulitis may at least partly depend on the microbiome [102]. In addition to its known effects of reducing bacterial overgrowth and improving the fecal mass, rifaximin may also exhibit an "eubiotic" effect, that is, the capacity of selectively promoting the growth of taxa with anti-inflammatory activity at the expense of pathobionts [103]. This capacity has been demonstrated in the context of Crohn's disease or cirrhosis with hepatic encephalopathy $[19,21]$, but studies in patients with diverticular disease are limited to very small case series.

In a group of patients with mixed diagnoses, including four subjects with SUDD, Ponziani et al. [23] were able to demonstrate that a 10-day trial with $400 \mathrm{mg}$ rifaximin tid was associated with significant, and potentially beneficial, changes in fecal microbiota composition, consisting of reduced representation of Roseburia, Veillonella, Streptococcus and Haemophilus. In another pilot study, the administration of different doses of rifaximin (800 mg/day or $1.6 \mathrm{~g} /$ day) for two weeks to seven female patients with SUDD resulted in the reduction of abundance of Akkermansia and different fecal metabolomics profiles, suggesting an active modulation of microbiome functionality [31].

The possible modifications in the intestinal microbiome induced by rifaximin are thus mostly subtle, but of great clinical interest. In fact, it does not affect the overall microbiome biodiversity [104], inducing selective depletion of a few taxa (Streptococcus and Veillonella) that may play a key role in the regulation of inflammation and mucosal barrier functionality [105]. These microbiome alterations do not affect systemic inflammation, but have relevance only for the gastrointestinal district [106]. Thus, the beneficial effects of rifaximin administration in SUDD and recurrent acute diverticulitis may depend on targeted antibiotic effects on the intestinal microbial communities, probably also inducing shifts in the functionality of bacteria [19, 21, 107]. Rifaximin also exhibits anti-inflammatory properties that are independent of the gut microbiota composition, making it a "pleiotropic" drug of great interest for the clinical management of diverticular disease and all other gastrointestinal disorders associated with dysbiosis [107]. These aspects deserve more attention in future studies enrolling patients with diverticular disease.

\section{CONCLUSIONS AND PERSPECTIVES}

The current scientific literature state-of-art suggests that the intestinal microbiome may be implied in several aspects of diverticulosis, ranging from risk factors, to progression into symptomatic diverticular disease, to treatment. The level of knowledge is anyway too incomplete to make microbiome science significantly modify the current clinical approach to patients with diverticula, with the only possible exception of rifaximin, which is considered in guidelines due to its eubiotic effect $[18,102]$.
However, in the foreseeable future, the management of many aspects of diverticular disease will take into consideration the intestinal microbiome. First, metagenomics is rapidly entering clinical practice, and many ongoing studies will contribute to define the reference standards for gut microbiota composition and the clinical significance of dysbiosis [4]. From this perspective, clinical profiling of the gut microbiota will allow a personalized management of several intestinal and extraintestinal diseases, including diverticulosis. A better knowledge of the effects of probiotics and pharmacological treatments on the gut microbiota composition and functionality will allow appropriate prescriptions, maximizing the benefits for patients.

Moreover, future studies should also focus on the role of the microbiota in patients with specific risk factors for diverticulosis, and on the correlation between the stage of diverticular disease and the composition/functionality of the microbiota. Recently, a novel endoscopic classification of diverticular disease, called DICA (Diverticular Inflammation and Complication Assessment), has entered clinical practice $[108,109]$. This exhibits an excellent correlation with patient outcomes, in terms of the risk of episodes of acute diverticulitis, and may be associated with specific intestinal microbiota alterations $[110,111]$. However, the correlation between diverticular disease staging and microbiota has been only marginally investigated to date, despite its potential relevance for the prescription of targeted personalized treatments.

In conclusion, the involvement of gut microbiota in the clinical course of diverticular disease is highly plausible, and clinically-oriented studies are needed to translate the microbiological concepts into clinical practice.

Conflicts of interest: A.N. and F.D.M. received a Congress Grant by Alpha Sigma in 2018. A.T. received a Congress Grant by Novo Nordisk in 2017. The authors have no further competing interests to declare.

Authors' contribution: A.T. and A.N. designed the study, contributed to paper selection for the review and wrote the manuscript. V.C. and C.T. contributed to the concept of the review and performed the database and paper selection. F.D.M. and T.M. contributed to the concept of the review and provided supervision.

\section{REFERENCES}

1. Young-Fadok TM. Clinical practice. Diverticulitis. N Engl J Med 2018;379:1635-1642. doi:10.1056/NEJMcp1800468

2. Violi A, Cambiè G, Miraglia C, et al. Epidemiology and risk factors for diverticular disease. Acta Biomed 2018;89(9-S):107-112. doi:10.23750/ abm.v89i9-S.7924

3. Ventura M, Turroni F, Canchaya C, Vaughan EE, O'Toole PW, van Sinderen D. Microbial diversity in the human intestine and novel insights from metagenomics. Front Biosci (Landmark Ed) 2009;14:32143221. doi: $10.2741 / 3445$

4. Ruppé E, Greub G, Schrenzel J. Messages from the first International Conference on Clinical Metagenomics (ICCMg). Microbes Infect 2017;19:223-228. doi:10.1016/j.micinf.2017.01.005

5. Belkaid Y, Hand TW. Role of the microbiota in immunity and inflammation. Cell 2014;157:121-141. doi:10.1016/j.cell.2014.03.011 
6. Ni J, Wu GD, Albenberg L, Tomov VT. Gut microbiota and IBD: causation or correlation? Nat Rev Gastroenterol Hepatol 2017;14:573584. doi:10.1038/nrgastro.2017.88

7. Nosho K, Sukawa Y, Adachi Y, et al. Association of Fusobacterium nucleatum with immunity and molecular alterations in colorectal cancer. World J Gastroenterol 2016;22:557-566. doi:10.3748/wjg.v22.i2.557

8. Nouvenne A, Ticinesi A, Tana C, et al. Digestive disorders and intestinal microbiota. Acta Biomed 2018;89(9-S):47-51. doi:10.23750/abm. v89i9-S.7912

9. Festi D, Schiumerini R, Eusebi LH, Marasco G, Taddia M, Colecchia A. Gut microbiota and metabolic syndrome. World J Gastroenterol 2014;20:16079-16094. doi:10.3748/wjg.v20.i43.16079

10. Ticinesi A, Tana C, Nouvenne A, Prati B, Lauretani F, Meschi T. Gu microbiota, cognitive frailty and dementia in older individuals: a systematic review. Clin Interv Aging 2018;13:1497-1511. doi:10.2147/ CIA.S139163

11. Ticinesi A, Milani C, Guerra A, et al. Understanding the gut-kidney axis in nephrolithiasis: an analysis of the gut microbiota composition and functionality of stone formers. Gut 2018;67:2097-2106. doi:10.1136/ gutjnl-2017-315734

12. Ticinesi A, Lauretani F, Milani C, et al. Aging gut microbiota at the cross-road between nutrition, physical frailty, and sarcopenia: is there a gut-muscle axis? Nutrients 2017;9:E1303. doi:10.3390/nu9121303

13. Ticinesi A, Tana C, Nouvenne A. The intestinal microbiome and its relevance for functionality in older persons. Curr Opin Clin Nutr Metab Care 2019;22:4-12. doi:10.1097/MCO.0000000000000521

14. Ticinesi A, Nouvenne A, Tana C, et al. The impact of intestinal microbiota on bio-medical research: definitions, techniques and physiology of a "new frontier". Acta Biomed 2018;89(9-S):52-59. doi:10.23750/abm.v89i9-S.7906

15. Ticinesi A, Lauretani F, Tana C, Nouvenne A, Ridolo E, Meschi T. Exercise and immune system as modulators of intestinal microbiome: implications for the gut-muscle axis hypothesis. Exerc Immunol Rev 2019;25:84-95.

16. Spiller RC. Changing views on diverticular disease: impact of aging, obesity, diet, and microbiota. Neurogastroenterol Motil 2015;27:305312. doi:10.1111/nmo.12526

17. Daniels L, Philipszoon LE, Boermeester MA. A hypothesis: important role for gut microbiota in the etiopathogenesis of diverticular disease. Dis Colon Rectum 2014;57:539-543. doi:10.1097/DCR.0000000000000078

18. Scarpignato C, Barbara G, Lanas A, Strate LL. Management of colonic diverticular disease in the third millennium: highlights from a symposium held during United European Gastroenterology Week 2017. Ther Adv Gastroenterol 2018;11:1756284818771305. doi:10.1177/1756284818771305

19. Pagliari D, Gambassi G, Piccirillo CA, Cianci R. The intricate link among gut "immunological niche", microbiota, and xenobiotics in intestinal pathology. Mediators Inflamm 2017;2017:8390595. doi:10.1155/2017/8390595

20. Skowron KB, Shogan BD, Rubin DT, Hyman NH. The new frontier: the intestinal microbiome and surgery. J Gastrointest Surg 2018;22:12771285. doi:10.1007/s11605-018-3744-7

21. Bajaj JS, Barbara G, DuPont HL, Mearin F, Gasbarrini A, Tack J. New concepts on intestinal microbiota and the role of non-absorbable antibiotics with special reference to rifaximin in digestive diseases. Dig Liver Dis 2018;50:741-749. doi:10.1016/j.dld.2018.04.020

22. Kvasnovsky CL, Leong LEX, Choo JM, et al. Clinical and symptom scores are significantly correlated with fecal microbiota features in patients with symptomatic uncomplicated diverticular disease: a pilot study. Eur J Gastroenterol Hepatol 2018;30:107-112. doi:10.1097/ MEG.0000000000000995

23. Ponziani FR, Scaldaferri F, Petito V, et al. The role of antibiotics in gut microbiota modulation: the eubiotic effects of rifaximin. Dig Dis 2016;34:269-278. doi:10.1159/000443361

24. Machiels K, Joossens M, Sabino J, et al. A decrease of the butyrateproducing species Roseburia hominis and Faecalibacterium prausnitzii defines dysbiosis in patients with ulcerative colitis. Gut 2014;63:12751283. doi:10.1136/gutjnl-2013-305799

25. Barbara G, Scaioli E, Barbaro MR, et al. Gut microbiota, metabolome and immune signatures in patients with uncomplicated diverticular disease. Gut 2017;66:1252-1261. doi:10.1136/gutjnl-2016-312377

26. Hayashi A, Sato T, Kamada N, et al. A single strain of Clostridium butyricum induces intestinal IL-10-producing macrophages to suppress acute experimental colitis in mice. Cell Host Microbe 2013;13:711-722. doi:10.1016/j.chom.2013.05.013

27. Atarashi $\mathrm{K}$, Tanoue $\mathrm{T}$, Oshima $\mathrm{K}$, et al. Treg induction by a rationally selected mixture of Clostridia strains from the human microbiota. Nature 2013;500:232-236. doi:10.1038/nature12331

28. Rodriguez-Nogales A, Algieri F, Garrido-Mesa J, et al. Differential intestinal anti-inflammatory effects of Lactobacillus fermentum and Lactobacillus salivarius in DSS mouse colitis: impact of microRNAs expression and microbiota composition. Mol Nutr Food Res 2017;61(11):1700144. doi:10.1002/mnfr.201700144

29. Daniels L, Budding AE, de Korte N, et al. Fecal microbiome analysis as a diagnostic test for diverticulitis. Eur J Clin Microbiol Infect Dis 2014;33:1927-1936. doi:10.1007/s10096-014-2162-3

30. Tursi A, Mastromarino P, Capobianco D, et al. Assessment of fecal microbiota and fecal metabolome in symptomatic uncomplicated diverticular disease of the colon. J Clin Gastroenterol 2016;50 Suppl 1:S9-S12. doi:10.1097/MCG.0000000000000626

31. Laghi L, Mastromarino P, Elisei W, et al. Impact of treatments on fecal microbiota and fecal metabolome in symptomatic uncomplicated diverticular disease of the colon: a pilot study. J Biol Regul Homeost Agents 2018;32:1421-1432.

32. Png CW, Lindén SK, Gilshenan KS, et al. Mucolytic bacteria with increased prevalence in IBD mucosa augment in vitro utilization of mucin by other bacteria. Am J Gastroenterol 2010;105:2420-2428. doi:10.1038/ajg.2010.281

33. Derrien M, Belzer C, de Vos WM. Akkermansia muciniphila and its role in regulating host functions. Microb Pathog 2017;106:171-181. doi:10.1016/j.micpath.2016.02.005

34. Lopetuso LR, Petito V, Graziani C, et al. Gut microbiota in health, diverticular disease, irritable bowel syndrome, and inflammatory bowel diseases: time for microbial marker of gastrointestinal disorders? Dig Dis 2018;36:56-65. doi:10.1159/000477205

35. Jiang F, Meng D, Weng M, et al. The symbiotic bacterial surface factor polysaccharide A on Bacteroides fragilis inhibits IL- $1 \beta$-induced inflammation in human fetal enterocytes via toll receptors 2 and 4 . PLoS One 2017;12:e0172738. doi:10.1371/journal.pone.0172738

36. Vandeputte D, Falony G, Vieira-Silva S, Tito RY, Joossens M, Raes J. Stool consistency is strongly associated with gut microbiota richness and composition, enterotypes and bacterial growth rates. Gut 2016;65:57-62. doi:10.1136/gutjnl-2015-309618

37. Vandeputte D, Kathagen G, D'hoe K, et al. Quantitative microbiome profiling links gut community variation to microbial load. Nature 2017;551:507-511. doi:10.1038/nature24460

38. Parthasarathy G, Chen J, Chen X, et al. Relationship between microbiota of the colonic mucosa vs feces and symptoms, colonic 
transit, and methane production in female patients with chronic constipation. Gastroenterology 2016;150:367-379.e1. doi:10.1053/j. gastro.2015.10.005

39. Carstens A, Roos A, Andreasson A, et al. Differential clustering of fecal and mucosa-associated microbiota in 'healthy' individuals. J Dig Dis 2018;19:745-752. doi:10.1111/1751-2980.12688

40. Spiller RC, Sloan TJ. Do diverticula provide a unique niche for microbiota which can lead to activation of the innate immune system? Gut 2017;66:1175-1176. doi:10.1136/gutjnl-2016-312928

41. Linninge C, Roth B, Erlanson-Albertsson C, Molin G, Toth E, Ohlsson B. Abundance of Enterobacteriaceae in the colon mucosa in diverticular disease. World J Gastrointest Pathophysiol 2018;9:18-27. doi:10.4291/ wjgp.v9.i1.18

42. Sassone-Corsi M, Nuccio SP, Liu H, et al Microcins mediate competition among Enterobacteriaceae in the inflamed gut. Nature 2016;540:280283. doi:10.1038/nature20557

43. Lupp C, Robertson ML, Wickham ME, et al. Host-mediated inflammation disrupts the intestinal microbiota and promotes the overgrowth of Enterobacteriaceae. Cell Host Microbe 2007;2:119-129. doi:10.1016/j.chom.2007.06.010

44. Schieffer KM, Sabey K, Wright JR, et al. The microbial ecosystem distinguishes chronically diseased tissue from adjacent tissue in the sigmoid colon of chronic, recurrent diverticulitis patients. Sci Rep 2017;7:8467. doi:10.1038/s41598-017-06787-8

45. Gueimonde M, Ouwehand A, Huhtinen H, Salminen E, Salminen S Qualitative and quantitative analyses of the bifidobacterial microbiota in the colonic mucosa of patients with colorectal cancer, diverticulitis and inflammatory bowel disease. World J Gastroenterol 2007;13:39853989. doi:10.3748/wjg.v13.i29.3985

46. Elian SD, Souza EL, Vieira AT, et al. Bifidobacterium longum subsp. Infantis BB-02 attenuates acute murine experimental model of inflammatory bowel disease. Benef Microbes 2015;6:277-286. doi:10.3920/BM2014.0070

47. Meng D, Zhu W, Ganguli K, Shi HN, Walker WA. Anti-inflammatory effects of Bifidobacterium longum subsp infantis secretions on fetal human enterocytes are mediated by TLR-4 receptors. Am J Physiol Gastrointest Liver Physiol 2016;311:G744-G753. doi:10.1152/ ajpgi.00090.2016

48. Jones RB, Fodor AA, Peery AF, et al. An aberrant microbiota is not strongly associated with incidental colonic diverticulosis. Sci Rep 2018;8:4951. doi:10.1038/s41598-018-23023-Z

49. Søreide K, Boermeester MA, Humes DJ, Velmahos GC. Acute colonic diverticulitis: modern understanding of pathomechanisms, risk factors, disease burden and severity. Scand J Gastroenterol 2016;51:1416-1422. doi:10.1080/00365521.2016.1218536

50. Scaioli E, Colecchia A, Marasco G, Schiumerini R, Festi D. Pathophysiology and therapeutic strategies for symptomatic uncomplicated diverticular disease of the colon. Dig Dis Sci 2016;61:673-683. doi:10.1007/s10620-015-3925-0

51. Tursi A, Mastromarino P, Capobianco D, et al. Urinary metabolic profiling and symptomatic uncomplicated diverticular disease of the colon. Clin Res Hepatol Gastroenterol 2017;41:344-346. doi:10.1016/j. clinre.2017.01.006

52. Sköldberg F, Olén O, Ekbom A, Schmidt PT. Appendectomy and risk of subsequent diverticular disease requiring hospitalization: a population-based case-control study. Dis Colon Rectum 2018;61:830 839. doi:10.1097/DCR.0000000000001086
53. Mandalia A, Kraft CS, Dhere T. Diverticulitis after fecal microbiota transplant for C. difficile infection. Am J Gastroenterol 2014;109:19561957. doi:10.1038/ajg.2014.350

54. Nascimbeni R, Casiraghi S, Cannatelli R, et al. Ulcerative colitis and the aging-related development of colonic diverticula. Int J Colorectal Dis 2018;33:1277-1283. doi:10.1007/s00384-018-3040-8

55. Bartels LE, Jepsen P, Tøttrup A, Vilstrup H, Dahlerup JF. Helicobacter pylori infection is associated with reduced prevalence of colonic diverticular disease. Helicobacter 2017;22:e12384. doi:10.1111/hel.12384

56. Scrivo R, Gerardi MC, Rutigliano I, et al. Polymyalgia rheumatica and diverticular disease: just two distinct age-related disorders or more? Results from a case-control study. Clin Rheumatol 2018;37:2573-2577. doi:10.1007/s10067-018-4137-8

57. Schmidt TSB, Raes J, Bork P. The human gut microbiome: from association to modulation. Cell 2018;172:1198-1215. doi:10.1016/j. cell.2018.02.044

58. O'Toole PW, Jeffery IB. Gut microbiota and aging. Science 2015;350:1214-1215. doi:10.1126/science.aac8469

59. Bischoff SC. Microbiota and aging. Curr Opin Clin Nutr Metab Care 2016;19:26-30. doi:10.1097/MCO.0000000000000242

60. Claesson MJ, Jeffery IB, Conde S, et al. Gut microbiota composition correlates with diet and health in the elderly. Nature 2012;488:178-184. doi:10.1038/nature11319

61. Haran JP, Bucci V, Dutta P, Ward D, McCormick B. The nursing home elder microbiome stability and associations with age, frailty, nutrition and physical location. J Med Microbiol 2018;67:40-51. doi:10.1099/ jmm.0.000640

62. Verdi S, Jackson MA, Beaumont M, et al. An investigation into physical frailty as a link between the gut microbiome and cognitive health. Front Aging Neurosci 2018;10:398. doi:10.3389/fnagi.2018.00398

63. Milani C, Ticinesi A, Gerritsen J, et al. Gut microbiota composition and Clostridium difficile infection in hospitalized elderly individuals: a metagenomic study. Sci Rep 2016;6:25945. doi:10.1038/srep25945

64. Santoro A, Ostan R, Candela M, et al. Gut microbiota changes in the extreme decades of human life: a focus on centenarians. Cell Mol Life Sci 2018;75:129-148. doi:10.1007/s00018-017-2674-y

65. Comparato G, Pilotto A, Franzè A, Franceschi M, Di Mario F. Diverticular disease in the elderly. Dig Dis 2007;25:151-159. doi:10.1159/000099480

66. Bharucha AE, Parthasarathy G, Ditah I, et al. Temporal trends in the incidence and natural history of diverticulitis: a population-based study. Am J Gastroenterol 2015;110:1589-1596. doi:10.1038/ajg.2015.302

67. Strate LS, Morris AM. Epidemiology, pathophysiology, and treatment of diverticulitis. Gastroenterology 2019;156:1282-1298.e1. doi:10.1053/j. gastro.2018.12.033

68. Heinritz SN, Weiss E, Eklund M, et al. Impact of a high-fat or high-fiber diet on intestinal microbiota and metabolic markers in a pig model. Nutrients 2016;8:E317. doi:10.3390/nu8050317

69. Dominianni C, Sinha R, Goedert JJ, et al. Sex, body mass index, and dietary fiber intake influence the human gut microbiome. PLoS One 2015;10:e0124599. doi:10.1371/journal.pone.0124599

70. Chen HM, Yu YN, Wang JL, et al. Decreased dietary fiber intake and structural alteration of gut microbiota in patients with advanced colorectal carcinoma. Am J Clin Nutr 2013;97:10441052. doi:10.3945/ajcn.112.046607

71. Cuervo A, Salazar N, Ruas-Madiedo P, Gueimonde M, González S. Fiber from a regular diet is directly associated with fecal short-chain fatty acid 
concentrations in the elderly. Nutr Res 2013;33:811-816. doi:10.1016/j. nutres.2013.05.016

72. Desai MS, Seekatz AM, Koropatkin NM, et al. A dietary fiber-deprived gut microbiota degrades the colonic mucus barrier and enhances pathogen susceptibility. Cell 2016;167:1339-1353. doi:10.1016/j. cell.2016.10.043

73. Tap J, Furet JP, Bensaada M, et al. Gut microbiota richness promotes its stability upon increased dietary fibre intake in healthy adults. Environ Microbiol 2015;17:4954-4964. doi:10.1111/1462-2920.13006

74. Chung WS, Walker AW, Louis P, et al. Modulation of the human gut microbiota by dietary fibres occurs at the species level. BMC Biol 2016;14:3. doi:10.1186/s12915-015-0224-3

75. Macia L, Tan J, Vieira AT, et al. Metabolite-sensing receptors GPR43 and GPR109A facilitate dietary fibre-induced gut homeostasis through regulation of the inflammasome. Nat Commun 2015;6:6734. doi:10.1038/ncomms7734

76. Ma N, Tian Y, Wu Y, Ma X. Contributions of the interaction between dietary protein and gut microbiota to intestinal health. Curr Protein Pept Sci 2017;18:795-808. doi:10.2174/1389203718666170216153505

77. Qi X, Wu W, Guo M, et al. Rice- or pork-based diets with similar calorie and content result in different rat gut microbiota. Int J Food Sci Nutr 2017;68:829-839. doi:10.1080/09637486.2017.1301889

78. Zhu Y, Shi X, Lin X, et al. Beef, chicken, and soy proteins in diets induce different gut microbiota and metabolites in rats. Front Microbiol 2017;8:1395. doi:10.3389/fmicb.2017.01395

79. Engen PA, Green SJ, Voigt RM, Forsyth CB, Keshavarzian A. The gastrointestinal microbiome: alcohol effects on the composition of intestinal microbiota. Alcohol Res 2015;37:223-236.

80. Bermon S, Petriz B, Kajeniene A, Prestes J, Castell L, Franco OL. The microbiota: an exercise immunology perspective. Exerc Immunol Rev 2015;21:70-79.

81. O'Sullivan O, Cronin O, Clarke SF, et al. Exercise and the microbiota. Gut Microbes 2015;6:131-136. doi:10.1080/19490976.2015.1011875

82. Allen JM, Mailing LJ, Niemiro GM, et al. Exercise alters gut microbiota composition and function in lean and obese humans. Med Sci Sports Exerc 2018;50:747-757. doi:10.1249/MSS.0000000000001495

83. Barton W, Penney NC, Cronin O, et al. The microbiome of professional athletes differs from that of more sedentary subjects in composition and particularly at the functional metabolic level. Gut 2018;67:625-633. doi:10.1136/gutjnl-2016-313627

84. Dao MC, Clément K. Gut microbiota and obesity: concepts relevant to clinical care. Eur J Intern Med 2018;48:18-24. doi:10.1016/j. ejim.2017.10.005

85. Menni C, Jackson MA, Pallister T, Steves CJ, Spector TD, Valdes AM. Gut microbiome diversity and high-fibre intake are related to lower long-term weight gain. Int J Obes 2017;41:1099-1105. doi:10.1038/ijo.2017.66

86. Williams PT. Incident diverticular disease is inversely related to vigorous physical activity. Med Sci Sports Exerc 2009;41:1042-1047. doi:10.1249/ MSS.0b013e318192d02d

87. Rosemar A, Angerås U, Rosengren A. Body mass index and diverticular disease: a 28-year follow-up study in men. Dis Colon Rectum 2008;51:450-455. doi:10.1007/s10350-007-9172-5

88. Mancabelli L, Milani C, Lugli GA, et al. Unveiling the gut microbiota composition and functionality associated with constipation through metagenomic analyses. Sci Rep 2017;7:9879. doi:10.1038/s41598-01710663-w

89. de Meij TG, de Groot EF, Eck A, et al. Characterization of microbiota in children with chronic functional constipation. PLoS One 2016;11:e0164731. doi:10.1371/journal.pone.0164731
90. Cao H, Liu X, An Y, et al. Dysbiosis contributes to chronic constipation development via regulation of serotonin transporter in the intestine. Sci Rep 2017;7:10322. doi:10.1038/s41598-017-10835-8

91. Sakuta H, Suzuki T. Prevalence rates of type 2 diabetes and hypertension are elevated among middle-aged Japanese men with colonic diverticulum. Environ Health Prev Med 2007;12:97-100. doi:10.1007/BF02898156

92. Yang F, Zheng Y, Jiang X, et al. Sex differences in risk factors of uncomplicated colonic diverticulosis in a metropolitan area from Northern China. Sci Rep 2018;8:138. doi:10.1038/s41598-017-18517-1

93. Lenti MV, Pasina L, Cococcia S, et al. Mortality rate and risk factors for gastrointestinal bleeding in elderly patients. Eur J Intern Med 2019;51:54-61. doi:10.1016/j.ejim.2018.11.003

94. Ticinesi A, Milani C, Lauretani F, et al. Gut microbiota composition is associated with polypharmacy in elderly hospitalized patients. Sci Rep 2017;7:11102. doi:10.1038/s41598-017-10734-y

95. Jackson MA, Verdi S, Maxan ME, et al. Gut microbiota associations with common diseases and prescription medications in a population-based cohort. Nat Commun 2018;9:2655. doi:10.1038/s41467-018-05184-7

96. Yuhara H, Corley DA, Nakahara F, et al. Aspirin and non-aspirin NSAIDs increase risk of colonic diverticular bleeding: a systematic review and meta-analysis. J Gastroenterol 2014;49:992-1000. doi:10.1007/s00535-013-0905-Z

97. Scarpignato C, Bertelé A, Tursi A. Probiotics for the treatment of symptomatic uncomplicated diverticular disease. Rationale and current evidence. J Clin Gastroenterol 2016;50:S70-S73. doi:10.1097/ MCG.0000000000000641

98. Lahner E, Annibale B. Probiotics and diverticular disease: evidence based? J Clin Gastroenterol 2016;50 Suppl 2:S159-S160. doi:10.1097/ MCG.0000000000000684

99. Lahner E, Bellisario C, Hassan C, Zullo A, Esposito G, Annibale B. Probiotics in the treatment of diverticular disease. A systematic review. J Gastrointestin Liver Dis 2016;25:79-86. doi:10.15403/ jgld.2014.1121.251.srw

100. Ojetti V, Petruzziello C, Cardone S, et al. The use of probiotics in different phases of diverticular disease. Rev Recent Clin Trial 2018;13:89-96. do i: $10.2174 / 1574887113666180402143140$

101. Lamiki P, Tsuchiya J, Pathak S, et al. Probiotics in diverticular disease of the colon: an open label study. J Gastrointestin Liver Dis 2010;19:31-36.

102. Lanas A, Abad-Baroja D, Lanas-Gimeno A. Progress and challenges in the management of diverticular disease: which treatment? Therap Adv Gastroenterol 2018;11:1756284818789055 doi: $10.1177 / 1756284818789055$

103. Ponziani FR, Zocco MA, D’Aversa M, Pompili M, Gasbarrini A. Eubiotic properties of rifaximin: disruption of the traditional concepts in gut microbiota modulation. World J Gastroenterol 2017;23:4491-4499. doi:10.3748/wjg.v23.i25.4491

104. Kaji K, Takaya H, Saikawa S, et al. Rifaximin ameliorates hepatic encephalopathy and endotoxinemia without affecting the gut microbiome diversity. World J Gastroenterol 2017;23:8355-8366. doi:10.3748/wjg.v23.i47.8355

105. Kawaguchi T, Suzuki F, Imamura M, et al. Rifaximin-altered gut microbiota components associated with liver/neuropsychological functions in patients with hepatic encephalopathy: an exploratory data analysis of phase II/III clinical trials. Hepatol Res 2019;49:404-418. doi:10.1111/hepr.13300

106. Jørgensen SF, Macpherson ME, Bjørnetrø T, et al. Rifaximin alters gut microbiota profile, but does not affect systemic inflammation - a randomized controlled trial in common variable immunodeficiency. Sci Rep 2019;9:167. doi:10.1038/s41598-018-35367-7 
107. Tursi A, Scarpignato C, Brandimarte G, Di Mario F, Lanas A. Rifaximin for the management of colonic diverticular disease: far beyond a simple antibiotic. J Gastrointestin Liver Dis 2018;27:351-355. doi:10.1540./ jgld.2014.1121.274.rif

108. Tursi A, Brandimarte G, Di Mario F, et al. Development and validation of an endoscopic classification of diverticular disease of the colon: the DICA Classification. Dig Dis 2015;33:68-76. doi:10.1159/000366039

109. Cambiè G, Violi A, Miraglia C, et al. Development and usefulness of the new endoscopic classification: DICA. Acta Biomed 2018;89(Suppl 9):113-118. doi:10.23750/abm.v89i9-S.7922
110. Tursi A, Brandimarte G, Di Mario F, et al. Predictive value of the Diverticular Inflammation and Complication Assessment (DICA) endoscopic classification on the outcome of diverticular disease of the colon: an international study. United European Gastroenterol J 2016;4:604-613. doi:10.1177/2050640615617636

111. Tursi A, Elisei W, Picchio M, et al. Impact of diverticular inflammation and complication assessment classification on the burden of medical therapies in preventing diverticular disease complications in Italy. Ann Transl Med 2017;5:320. doi:10.21037/ atm.2017.06.39 